Artículo científico

Volumen 30(3):721-732. Septiembre-diciembre, 2019 e-ISSN 2215-3608, doi:10.15517/am.v30i3.34471

http://www.revistas.ucr.ac.cr/index.php/agromeso

\title{
Productividad y calidad de espárragos verdes masculinos en Azul, Buenos Aires, Argentina ${ }^{1}$
}

\section{Productivity and quality of male green asparagus in Buenos Aires, Argentina}

\author{
Ana María Castagnino², Karina Elizabeth Díaz ${ }^{3}$, Andrea Paola Guisolis ${ }^{3}$, William John Rogers ${ }^{4}$, \\ Agostino Falavigna ${ }^{5}$, María Belén Rosini ${ }^{3}$
}

1 Recepción: 6 de setiembre, 2018. Aceptación: 13 de marzo, 2019. Este trabajo formó parte del Programa de Investigación y Transferencia Tecnológica 03A/228 "Cadena Esparrago bajo un enfoque sistémico" del Centro Regional de Estudio Sistémico de Cadenas Agroalimentarios (CRESCA), Facultad de Agronomía (FAA), Universidad Nacional del Centro de la Prov. de Bs. As. (UNCPBA), Argentina, y Proyecto CIAC-940186.

2 Universidad Nacional del Centro de la Provincia de Buenos Aires (CRESCA, FAA, UNICEN), y Pontificia Universidad Católica Argentina (UCA). CC 47 (7300) Azul, Argentina. amc@faa.unicen.edu.ar (https://orcid.org/0000-0002-5067-0964).

3 Universidad Nacional del Centro de la Provincia de Buenos Aires (CRESCA, FAA, UNICEN) CC 47 (7300) Azul, Argentina. kardiaz@faa. unicen.edu.ar, andreaguisolis@hotmail.com, belenrosini@yahoo.com.ar

4 Universidad Nacional del Centro de la Provincia de Buenos Aires (CRESCA, FAA, UNICEN) CC 47 (7300), Comisión de Investigaciones Científicas-Laboratorio de Biología Funcional y Biotecnología (CIC-BIOLAB AZUL), y Consejo Nacional de Investigaciones Científicas y Técnicas-Instituto de Investigaciones en Biodiversidad y Biotecnología (CONICET-INBIOTEC). Azul, Argentina. rogers@faa.unicen.edu.ar

5 Consiglio della Ricerca Agraria (CRA)- Unità di Montanaso Lombardo, Lodi. Italia. agostino.falavigna@entecra.it

\section{Resumen}

Introducción. En el cultivo de espárrago, por tratarse de una alternativa productiva perenne, se requiere conocer la respuesta de distintos genotipos a la zona en la que se pretende introducirlos. Objetivo. El objetivo del presente trabajo fue determinar la productividad de siete híbridos masculinos italianos y un testigo americano de espárrago verde de una plantación adulta en su etapa de productividad estable. Materiales y métodos. El estudio se llevó a cabo en Azul, Argentina (19/8/2016-17/11/2016), donde se evaluaron los genotipos: Italo, Zeno, Eros, Ercole, H-668, Marte, Giove y el testigo UC-157. Se compararon plantines grandes y chicos, y dos largos de corte $(22$ y $17 \mathrm{~cm})$. Las variables fueron: productividad comercial total fresca de turiones largos y cortos, número de turiones comerciales totales largos y cortos, peso medio de turiones largos y cortos, distribución de calibres: muy pequeño (asparagina) (A $<6 \mathrm{~mm})$, pequeño $(\mathrm{S}$; 6-9 mm), mediano (M; 9-12 mm), grande (L; 12-16 mm), extra grande (XL; 16-18 mm) y gigante $(\mathrm{J} ;>18 \mathrm{~mm})$, y los defectos: turiones muy cortos, turiones espigados, turiones con daño de plagas y otros como turiones torcidos, dobles planos o enfermos. Los datos se analizaron por ANOVA $(\mathrm{p} \geq 0,05)$ y la prueba LSD restringida de Fisher. Resultados. La producción comercial promedio de primera calidad lograda con los dos largos de cosecha fue 4,41 t ha ${ }^{-1}(37 \%$ turiones cortos y $63 \%$ largos). Por planta se obtuvieron diecisiete turiones (44\% turiones cortos y $56 \%$ largos). En número de turiones comerciales totales (267 189 turiones ha-1), Ercole, Giove, UC-157, Eros y H-668, superaron la media. Los genotipos de mayor productividad comercial fueron Giove y Ercole, mientras que por número de turiones, Eros y H-668. El principal defecto fue el espigado (93\%). Conclusiones. A fin de optimizar el rendimiento comercial y minimizar el defecto de espigado se sugiere cosechar diariamente, y emplear dos largos de corte, para incrementar el porcentaje de turiones comerciales.

Palabras clave: hortalizas de tallo, Asparagus officinalis, rendimiento de cultivos, calidad de los alimentos. 


\begin{abstract}
Introduction. In asparagus cultivation, as it is a perennial productive alternative, evaluation is required to know the response of different genotypes to the area in which are being introduced. Objective. The aim of the present work was to determine the productivity of seven Italian male hybrids and an American control of green asparagus from an adult plantation in its stage of stable productivity. Materials and methods. The study was carried out in Azul, Argentina (19/8/2016-17/11/2016), where the following genotypes were evaluated: Italo, Zeno, Eros, Ercole, H-668, Marte, Giove, and the control UC-157. Large and small seedling and two cutting lengths $(22$ and $17 \mathrm{~cm})$ were compared. The variables were: total fresh commercial productivity of long and short spears, number of long and short total commercial spears, mean weight of long and short spears, calibre distribution: very small (asparagina) $($ A $<6$ $\mathrm{mm})$, small (S; 6-9 mm), medium (M; 9-12 mm), large (L; 12-16 mm), extra-large (XL; 16-18 mm) and giant (J; >18 $\mathrm{mm}$ ), and the defects: very short spears, sprouted spears, plague damaged spears and other such as twisted, double or diseased spears. Data were analysed by ANOVA $(\mathrm{p} \geq 0.05)$ and Fisher's restricted LSD test. Results. The average first quality commercial production achieved with both harvested lengths was $4.41 \mathrm{t} \mathrm{ha}^{-1}$ (37\% short and $63 \%$ long spears). Seventeen spears were obtained per plant (44\% short and $56 \%$ long spears). In number of total commercial spear (267,189 spears ha $\left.{ }^{-1}\right)$, Ercole, Giove, Eros, and H-668 exceeded the mean. The genotypes with the highest commercial productivity were Giove and Ercole, while by number of spears Eros and H-668. The main defect was sprouting (93\%). Conclusions. In order to optimize the commercial yield and minimize the sprouting defect, it is suggested to harvest daily and to use two cutting lengths to increase commercial spear percentage.
\end{abstract}

Keywords: steam vegetables, Asparagus officinalis, crop yield, food quality.

\title{
Introducción
}

En Argentina, el espárrago (Asparagus officinalis var. altilis) representa una alternativa agrícola de diversificación productiva perenne, de la que existen diferentes genotipos en el mercado. Estos incluyen híbridos enteramente masculinos cuya productividad es más homogénea y de mayor calidad, sin embargo, resultaría conveniente evaluarlos en distintas condiciones de cultivo. Asimismo, resulta conveniente conocer su tendencia respecto del grosor (calibres) y largo comercial.

El espárrago es una especie de consumo estacional que participa en la dieta de un limitado segmento poblacional en los países latinoamericanos, en donde pocas personas conocen sus características y los beneficios de su consumo. A nivel global, por el contrario, es muy valorado por sus características organolépticas y los beneficios nutricionales. Se ha comprobado y difundido que presenta una elevada relación de nutrientes / calorías (Vázquez-Rowe et al., 2016) y contenido en antioxidantes, como por ejemplo ácido ascórbico o glutatión (Sun et al., 2007; Kim et al., 2009; Wang et al., 2011), flavonoides (principalmente rutina) y otros compuestos fenólicos. Dicha actividad antioxidante, presente también en muchos de los vegetales comúnmente consumidos (Makris y Rossiter, 2001; Sun et al., 2007; Chen et al., 2015), puede ayudar a prevenir el envejecimiento y enfermedades (Vázquez-Rowe et al., 2016), y a disminuir el riesgo de enfermedades degenerativas y ciertos tipos de cáncer, por la reducción del estrés oxidativo y la inhibición de la oxidación de las macromoléculas (Soobrattee et al., 2005).

La producción y consumo de hortalizas no tradicionales, como los espárragos, resulta recomendable para la salud, especialmente en países como Argentina que actualmente tienen la necesidad de incrementar la participación de hortalizas en la dieta, ya que, según el informe realizado por la Organización de las Naciones Unidas para la Alimentación y la Agricultura, la Organización Panamerica de la Salud, el Programa Mundial de Alimentos y el Fondo de las Naciones Unidas para la Infancia (FAO et al., 2018), cuenta con la mayor tasa de obesidad de América Latina $(28,3 \%)$ y un índice de sobrepeso que supera el $60 \%$ de la población, y que además, presenta serias carencias nutricionales, en gran parte por el bajo consumo en cantidad y diversidad de hortalizas. Según 
el informe OMS y FAO (2003), la ingesta recomendada de hortalizas y frutas es de $400 \mathrm{~g}$ diarios, y según las guías alimentarias para la población argentina (MSA, s.f.) elaboradas por la Asociación Argentina de Dietistas y Nutricionistas Dietistas (AADYND) en el año 2000 y actualizadas en 2016, se recomienda consumir $400 \mathrm{~g}$ de hortalizas no feculentas (excluyendo papa, batata, etc.) y $300 \mathrm{~g}$ de frutas, es decir, un total de $700 \mathrm{~g}$.

El espárrago, a nivel mundial, es considerado una hortaliza gourmet, cada vez más consumida en los últimos años, debido no solo a su valor nutricional sino también por su sabor, el cual es más pronunciado en espárragos verdes, incluidos en el presente estudio. Este es un producto perecedero con una vida útil corta después de la cosecha, debido a su alta tasa de respiración que conduce rápidamente a senescencia (Techavuthiporn y Boonyaritthongchai, 2016), por lo que, se requiere un acondicionamiento inmediato luego de la cosecha que incluya corte, lavado, selección y calibrado, a fin de cuantificar su rendimiento comercial. En dicho proceso, son diversos los problemas que pueden derivar en un descarte de un porcentaje de la producción, como lo son turiones espigados, torcidos, malformados, muy cortos, con daños de plagas, entre otros. A estos se le suman la pérdida de calidad durante la poscosecha como la marchitez de los turiones, endurecimiento como resultado de su lignificación (Bhowmik et al., 2001), degradación de la clorofila (Albanese et al., 2007), y cambios en el contenido de ácido orgánico y azúcar (Bhowmik et al., 2000). Es así que resulta prioritario una adecuada elección del genotipo a cultivar, tendiente a determinar aquellos que presenten menor proporción de defectos, motivo de descarte. Para un mejor posicionamiento del producto en el mercado es necesario que los turiones sean verdes en al menos $80 \%$ de su largo, que estén presentados turgentes, bien formados y sanos (SAGPyA, 2007). Asimismo, la presencia de plagas animales y enfermedades pueden afectar tanto estética como sanitariamente la presentación de los espárragos, por lo que, obtener información respecto de la incidencia de agentes animales que afecten negativamente la calidad comercial de los turiones, resulta de fundamental importancia. En el espárrago verde, el segmento apical presenta una composición que incluye mayores valores de compuestos antioxidantes como ascorbato, clorofila a y b, carotenoides, antocianinas y lignina; mientras que el segmento basal contiene más azúcares y almidón, por lo que resulta importante, a nivel nutricional, el consumo tanto de partes apicales como basales (Mastropasqua, 2016). Actualmente, existe una tendencia a la diversificación de presentaciones en función de la demanda del mercado, que incluye la comercialización de turiones cortos (de menos de los $22 \mathrm{~cm}$ vendidos de manera tradicional), generalmente de entre 10 y $17 \mathrm{~cm}$, por lo que en dicho caso, las puntas de espárrago presentan un menor contenido de azúcares y almidón (Mastropasqua, 2016), y menor fibrosidad. El rápido deterioro del espárrago en almacenamiento ha sido un problema importante en la exhibición minorista en los supermercados, ya que los turiones almacenados tienen una vida útil más corta (Lill et al., 1990); con respecto a esto, Bhowmik et al. (2000) indicaron que el período de almacenamiento afectó los cambios de textura y composición de puntas de espárragos después de una simulación de venta minorista. Aunque mucha investigación se ha centrado en mejorar el almacenamiento de espárragos, pocos informes documentan la importancia del comportamiento de cada genotipo en las distintas etapas de la cadena y su incidencia en los cambios que generalmente ocurren durante las simulaciones de almacenamiento, transporte y exhibición en súper e híper mercados hasta su consumo.

Las características alimenticias saludables y las particularidades del cultivo del espárrago, han motivado que el consumo a nivel global presente una tendencia creciente (Santos, 2011), y representa una oportunidad productiva para los países del hemisferio sur por la contraestación respecto a los países centrales, y para su comercialización tanto a nivel interno como externo, lo que hace necesario la evaluación de aquellos genotipos que puedan adaptarse mejor a cada ambiente de cultivo. La superficie global total actual del cultivo de espárragos es de 180000 ha, destacándose China (70 000 ha), Perú (25 000 ha), Alemania (22 000 ha) y México (16 000 ha) (Limgroup, 2016). En Argentina, el $93 \%$ del volumen comercializado anualmente corresponde al período de septiembre a diciembre, lo que demuestra la marcada estacionalidad de la producción. Respecto de los aspectos técnicos del cultivo, la vida económica se considera que son diez años, llegando al máximo de producción a los cuatro o cinco años (Marina et al., 2010), no obstante, el cultivo en producción puede durar varias décadas. Los rendimientos que se obtienen son 
muy variables y están condicionados por la edad de la plantación, la variedad, las condiciones climáticas, el manejo del lote y el origen de la semilla empleada (Marina et al., 2010; Novella, 2017). El ciclo de vida del espárrago puede ser subdividido en las siguientes fases: 1) crecimiento temprano (primeros dos años), caracterizada por un fuerte desarrollo vegetativo; 2) productividad creciente ( $3-4^{\circ}$ año), que corresponde a los dos primeros años de cosecha; 3) productividad estable (4-12 año); y 4) productividad decreciente (12-20 ${ }^{\circ}$ año) (Falavigna, 2006). En las evaluaciones de esta especie, como testigo deben incluirse híbridos o variedades de las cuales se conozcan las características productivas y cualitativas. Los productores argentinos de espárrago, tradicionalmente han cultivado el híbrido dioico americano UC-157 (Novella et al., 2017), incluido como testigo en el presente ensayo.

El objetivo del presente trabajo fue determinar la productividad de siete híbridos masculinos italianos y un testigo americano de espárrago verde de una plantación adulta en su etapa de productividad estable.

\section{Materiales y métodos}

El ensayo se realizó en Azul, en la Chacra de la Facultad de Agronomía de la Universidad Nacional del Centro de la Provincia de Buenos Aires (UNCPBA) (36 $48^{\prime}$ lat. S y 59 51' long. O), Argentina, cuya plantación definitiva se efectuó el 22/11/2006. La evaluación se efectuó al noveno año productivo, en el que se realizaron 39 cosechas en el periodo comprendido entre el 19/8/2016 y el 17/11/2016 (etapa de productividad estable), con una frecuencia de cosecha de día por medio. El ensayo se realizó sobre un suelo argiudol típico sin impedimentos en el perfil, con un adecuado contenido de materia orgánica $(3,74 \%)$, con un pH levemente ácido, conductividad eléctrica normal $\left(143,5 \mu \mathrm{S} \mathrm{cm}^{-1}\right), \mathrm{N}$ (nitrógeno) total $(0,183 \%)$ moderado a bien provisto y bien provisto de fósforo $(33,07 \mathrm{ppm})$, de acuerdo con el análisis efectuado en el Laboratorio de Análisis de Suelo de la Facultad de Agronomía (UNCPBA). La zona de estudio cuenta con clima templado, una temperatura media anual de $15{ }^{\circ} \mathrm{C}$, con promedios en verano de $22{ }^{\circ} \mathrm{C}$ y en invierno de $8{ }^{\circ} \mathrm{C}$, y un promedio de precipitaciones de $960 \mathrm{~mm}$ anuales.

El diseño experimental fue de bloques al azar con cuatro repeticiones, la parcela fue la unidad de muestreo, constituida cada una por veintisiete plantas. La superficie de cada parcela fue de $10 \mathrm{~m}^{2}$, con un largo de $7 \mathrm{~m}$ y un ancho de $1,4 \mathrm{~m}$.

La superficie total del ensayo fue de $1700 \mathrm{~m}^{2}$, con un ancho total de $34 \mathrm{~m}$ ( 17 surcos a $1,4 \mathrm{~m}$ entre surcos y 5 $\mathrm{m}$ a ambos lados de caminos); y un largo de $50 \mathrm{~m}$ (7 m de largo de cada uno de los cuatro bloques, tres caminos internos de $4 \mathrm{~m}$ cada uno y $5 \mathrm{~m}$ cabeceras en ambos lados). La superficie real de evaluación fue de $588 \mathrm{~m}^{2}$, considerando la superficie de los cuatro bloques de cultivo y sus borduras laterales.

La profundidad de plantación fue de $0,25 \mathrm{~m}$ y la densidad fue de 23809 plantas ha $^{-1}$, correspondiente a un marco de plantación de 1,4 m entre líneas y 0,3 m entre plantas.

Previo a la plantación se realizó una fertilización de fondo en banda, con $200 \mathrm{~kg} \mathrm{ha}^{-1}$ de fosfato diamónico y, posteriormente en cobertura, durante el verano, se efectuaron aplicaciones parcializadas de urea en banda, con dosis acorde a cada momento del ciclo de cultivo.

Se controlaron las malezas con una combinación de labores mecánicas, químicas y manuales, mediante remociones con motocultivador, trabajos manuales en las hileras de cultivo y aplicaciones de herbicidas: el primer año con Linurón al 37,6 \% a razón de 1,2 1 ha $^{-1}$ y a partir del segundo desde la plantación con Metribuzín 0,5 $\mathrm{kg} \mathrm{ha}^{-1}$ al 35 $\%$ y Pendimetalín 2,51 ha-1 al 31,7 \%, en mezcla en pre-emergencia según las recomendaciones de Falavigna (2004).

En cuanto al riego, se regó el año de la plantación por surco (2006) y los dos siguientes años (2007 y 2008) por goteo. Posteriormente, el cultivo se manejó en secano sin riego, dado que la zona cuenta con aproximadamente 900 mm por año, y coinciden los máximos registros con los periodos de cosecha y vegetativo del cultivo (Requesens, 2011). Los genotipos italianos masculinos evaluados fueron: Italo, Zeno, Eros, Ercole, H-668, Marte y Giove, y el testigo americano fue UC-157. Las características de los genotipos según Falavigna (2004; 2006) son las siguientes: 
- Italo: primer híbrido íntegramente masculino producido en Italia, adaptado a ambientes cálidos y áridos del Mediterráneo, de turiones muy homogéneos con cerrado óptimo de brácteas que ha manifestado alto vigor en las plantas en terrenos infectados con Fusarium.

- Zeno: híbrido masculino, con esfumaduras antociánicas de calibre medio, adaptado a la producción de turiones tanto verdes como blancos, aunque mejor adaptado a blancos.

- Eros: enteramente masculino, caracterizado por una elevada productividad en su zona de origen (norte de Italia), de precocidad media, empleado tanto para la producción de turiones verdes como blancos. De elevado calibre (calibres jumbo, extra large y large), intensa coloración antociánica y elevada dimensión de las brácteas, las que permanecen adheridas a la punta del turión, aún cuando superan los $20 \mathrm{~cm}$ de altura. Parcialmente resistente a roya y sensible a Stemphylium sp.

- Ercole: caracterizado por su elevada productividad, aunque más precoz que Eros, de color verde brillante.

- H-668: híbrido experimental que al momento de la plantación aún no era comercial.

- Marte: híbrido enteramente masculino, que presenta una elevada uniformidad fenotípica, con parcial resistencia a roya, tolerante a Fusarium sp. y sensible a Stemphylium sp. De productividad y calibre medio y uniforme, adaptado a la producción de turiones blancos y verdes. De color de fondo verde oscuro y brácteas violáceas bien cerradas.

- Giove: híbrido enteramente masculino caracterizado por su elevado calibre y tendencia a buen cierre de brácteas.

- UC 157 (testigo): híbrido dioico, muy precoz, con turiones de calibre medio, con brácteas cerradas, aún en condiciones de cosecha con altas temperaturas. Tradicionalmente cultivado en Argentina, Colombia, Perú, Ecuador y Chile. Ha demostrado gran adaptación, altos rendimientos y una producción temprana de turiones uniformes en color y tamaño.

El principal motivo de la realización del presente ensayo se debe a las ventajas de cultivar dichos genotipos, respecto de los dioicos (con $50 \%$ de plantas femeninas y $50 \%$ de plantas masculinas) con base en que las plantas masculinas son más productivas, longevas y tolerantes a enfermedades con respecto a las femeninas. Además, los turiones son más homogéneos siendo eliminados todos los dimorfismos asociados a la expresión sexual, entre los que se destacan el calibre, que es mayor en las plantas femeninas (Falavigna, 2007).

Los turiones se cosecharon manualmente, cortando al ras del suelo con un corte transversal y neto, evitando el corte a bisel cuando los mismos tenían un largo superior a los $18 \mathrm{~cm}$, y fueron trasladados para su acondicionamiento en fresco en el laboratorio de procesado de la Facultad de Agronomía (UNCPBA). Una vez ingresados, se seleccionaron, cortaron, pesaron, contaron y calibraron (largo: $22 \mathrm{~cm}$, corto: $17 \mathrm{~cm}$ ), siguiendo las recomendaciones del protocolo de calidad de espárragos frescos (SAGPyA, 2007).

Las variables estudiadas fueron: productividad comercial total fresca (PCTF), productividad comercial fresca con dos largos de cortes: turiones largos (PC-L) cosechados a $22 \mathrm{~cm}$ y cortos (PC-C) a $17 \mathrm{~cm}$, número de turiones comerciales totales (NTCT) largos (NTC-L) y cortos (NTC-C), peso medio del turión (PPT) largo (PPT-L) y corto (PPT-C) y distribución de calibres (diámetro a $2 \mathrm{~cm}$ desde la base) en muy pequeño o asparagina $(\mathrm{A}<6 \mathrm{~mm})$, pequeño (S; 6 - $9 \mathrm{~mm}$ ), mediano (M; 9 - $12 \mathrm{~mm}$ ), largo (L; 12 - $16 \mathrm{~mm}$ ), extra largo (XL; 16 - $18 \mathrm{~mm}$ ) y muy grande o gigante $(\mathrm{J} ;>18 \mathrm{~mm})$, tal como indica el protocolo de calidad argentino (SAGPyA, 2007). Se contemplaron los defectos encontrados: turiones muy cortos (DC), espigados (DE), con daño de plagas (DP) y otros defectos como torcidos, dobles planos o enfermos (DO).

El análisis de los datos fue por ANOVA $(\mathrm{P} \geq 0,05)$ y la prueba LSD restringida de Fisher, la cual ofrece un grado de protección contra errores de Tipo I, sin perjudicar demasiado el poder de la prueba en la identificación de diferencias potenciales entre los genotipos de posible interés comercial (Snededor y Cochran, 1980). Para el análisis estadístico se utilizó el programa InfoStat (Di-Rienzo et al., 2018). 


\section{Resultados}

La producción comercial promedio de primera calidad, lograda en el noveno año productivo con dos largos de cosecha, fue de $4,41 \mathrm{t} \mathrm{ha}^{-1}$, lo que correspondió a un $37 \%$ turiones cortos y $63 \%$ largos; mientras que el número de turiones por planta fue de diecisiete unidades, con $44 \%$ de turiones cortos y $56 \%$ largos (Cuadro 1). En promedio el número de turiones comerciales producidos fue de 267189 por hectárea, donde Ercole, Giove, Eros y H-668 superaron la media (Cuadro 1).

En turiones comerciales cosechados de diferentes largos, en orden de importancia, se destacaron Giove, Testigo, H-668, Ercole y Eros (Cuadro 1), y los genotipos que superaron la media en turiones cosechados largos: Giove en $28 \%$, Ercole en $24 \%$, UC-157 en 19,6 \%, Eros en $10 \%$ y H-668 en 2,5 \% (Cuadro 1).

En el caso de los turiones cortos, los que requieren una presentación distinta a la tradicional en atados, cuatro genotipos superaron la media: H-668, Ercole, UC-157 y Eros (Cuadro 1).

Cuadro 1. Productividad de ocho genotipos de espárrago verde (Asparagus officinalis var. altilis) de una plantación en su noveno año productivo. Azul, Buenos Aires, Argentina. 2016.

Table 1. Productivity of eight green asparagus genotypes (Asparagus officinalis var. altilis) from a plantation in its ninth production year. Azul, Buenos Aires, Argentina. 2016.

\begin{tabular}{|c|c|c|c|c|c|c|}
\hline \multirow[t]{2}{*}{ Híbrido } & \multicolumn{3}{|c|}{ Peso fresco comercial $\left(t_{\text {ha-1 }}^{-1}\right)$} & \multicolumn{3}{|c|}{ Número de turiones comerciales (NTC ha') } \\
\hline & Cortos** & Largos** & Total & Cortos & Largos & Total \\
\hline Giove & $1,87^{\mathrm{a}}$ & $3,66^{\mathrm{a}}$ & $5,53^{a}$ & $116002^{a b}$ & $190499^{a}$ & $306501^{a}$ \\
\hline UC-157* & $1,84^{\text {ab }}$ & 3,47 ab & 5,32 ab & $126001 \mathrm{ab}$ & $178000^{\text {ab }}$ & $304005^{\mathrm{a}}$ \\
\hline H-668 & $1,79 \mathrm{abc}$ & 2,63 bc & 4,43 bc & $131500^{\mathrm{a}}$ & $152502^{\mathrm{bc}}$ & $283998^{a}$ \\
\hline Ercole & 1,74 abcd & 3,21 ab & $4,95 \mathrm{abc}$ & 126500 ab & 184540 ab & $310986^{a}$ \\
\hline Eros & 1,54 abcd & $2,92^{a b}$ & $4,46^{\mathrm{bc}}$ & $122000 \mathrm{ab}$ & $164498^{a b}$ & $286494^{a}$ \\
\hline Marte & $1,47^{\mathrm{bcd}}$ & $1,63^{\mathrm{d}}$ & $3,10^{\mathrm{d}}$ & $102001^{\mathrm{b}}$ & $90999^{\mathrm{d}}$ & $193011^{\mathrm{b}}$ \\
\hline Italo & $1,43^{\mathrm{cd}}$ & 2,81 ab & $4,24^{\mathrm{c}}$ & 107500 ab & $124001^{\mathrm{cd}}$ & $231504^{b}$ \\
\hline Zeno & $1,37^{\mathrm{d}}$ & $1,82^{\mathrm{cd}}$ & $3,22^{d}$ & 115499 ab & $105499^{d}$ & $221013^{\mathrm{b}}$ \\
\hline Promedio & 1,63 & 2,77 & 4,41 & 118375 & 148817 & 267189 \\
\hline
\end{tabular}

* testigo / * control.

** turiones largos cosechados a $22 \mathrm{~cm}$ y cortos a $17 \mathrm{~cm} / * *$ long spears harvested at $22 \mathrm{~cm}$ and short at $17 \mathrm{~cm}$.

En cuanto al peso promedio del turión (PPT), la media fue de $16,3 \mathrm{~g}$ turión $^{-1}$. Los cortos pesaron $14 \mathrm{~g}$ y los largos 18,6 g; se destacaron en el primer caso los híbridos Giove (16) y UC-157 (testigo) (15), y en largos Italo (23), Testigo (20) y Giove (19) (Cuadro 2).

Los genotipos Giove, Testigo y Ercole, se destacaron por su productividad comercial total (Cuadro 1), lo cual indica la conveniencia de su cultivo para la zona de estudio.

En turiones cortos los genotipos de mayor productividad, en orden de importancia, fueron: Giove, Testigo, H-668, Ercole y Eros; mientras que en turiones largos: Giove, Testigo, Ercole, Eros e Italo (Cuadro 1).

Respecto de la cantidad de turiones con los diferentes largos de corte, en general, los genotipos en estudio lograron mayor proporción de turiones largos, a excepción de Marte y Zeno (Cuadro 1). 
Cuadro 2. Peso promedio por turión, de diferentes largos de corte, de ocho genotipos de espárragos verdes (Asparagus officinalis var. altilis), obtenidos de una plantación de secano en su noveno año productivo. Azul, Buenos Aires, Argentina. 2016.

Table 2. Mean spear weight, of different cut lengths, of eight green asparagus genotypes (Asparagus officinalis var. altilis) obtained from a dry plantation in its ninth production year. Azul, Buenos Aires, Argentina. 2016.

\begin{tabular}{cccc}
\hline Híbrido & $\begin{array}{c}\text { Peso promedio turiones } \\
\text { cortos** }^{*}\end{array}$ & $\begin{array}{c}\text { Peso promedio turiones } \\
\text { largos** }\end{array}$ & Peso promedio por turión \\
\cline { 2 - 4 } g turion $^{-1}$ & \\
\hline Giove & 16,13 & 19,23 & 17,68 \\
Uc-157* & 14,64 & 19,52 & 17,08 \\
H668 & 13,64 & 17,26 & 15,45 \\
Ercole & 13,73 & 17,38 & 15,55 \\
Eros & 12,63 & 17,76 & 15,19 \\
Marte & 14,46 & 17,88 & 16,17 \\
Italo & 13,31 & 22,64 & 17,97 \\
Zeno & 11,89 & 17,25 & 14,57 \\
\hline Promedio & 13,80 & 18,62 & 16,21 \\
\hline
\end{tabular}

PPTC: peso promedio del turión corto; PPTL: peso promedio del turión largo / PPTC: mean short spear weight; PPTL: mean long spear weight.

* testigo / * control.

** turiones largos (PC-L) cosechados a $22 \mathrm{~cm}$ y cortos $(\mathrm{PC}-\mathrm{C})$ a $17 \mathrm{~cm} / * *$ long spears harvested at $22 \mathrm{~cm}$ and short at $17 \mathrm{~cm}$.

En turiones comerciales cosechados de diferentes largos, en orden de importancia, se destacaron: Giove, Testigo, H-668, Ercole, Eros, Marte, Italo y Zeno (Cuadro 1), y los genotipos que superaron la media en turiones cosechados largos: Giove en $28 \%$, Ercole en $24 \%$, UC-157 en 19,6 \%, Eros en $10 \%$ y H-668 en 2,5 \% (Cuadro 1).

En el caso de los turiones cortos, los que requieren una presentación distinta a la tradicional en atados, cuatro genotipos superaron la media: H-668, Ercole, UC-157 y Eros (Cuadro 1). En distribución de calibres el $41 \%$ de los turiones procesados correspondió a elevados calibres (J, XL y L), mientras que el $59 \%$ a los inferiores (M, S y Asp.) (Cuadro 3).

Los híbridos Giove y UC-157, presentaron una distribución homogénea en producción de elevados calibres (50 \%) y bajos (50\%), mientras que en el caso de los genotipos: Marte, Italo, Eros, Ercole y H-668, se obtuvo el $40 \%$ aproximadamente, y en Zeno $30 \%$ (Cuadro 3).

El principal defecto encontrado fue el espigado (93\%), posiblemente debido a la frecuencia de cosecha utilizada (Cuadro 4). El defecto que le siguió en importancia fue el denominado "otros defectos", donde se incluyeron turiones torcidos, huecos, espiralados, dobles, etc. Los turiones que debieron cosecharse muy cortos por su prematura tendencia al espigado representaron $36 \%$ de defectos, y por último, el daño de plagas resultó en tan solo $3 \%$ (Cuadro 4). El defecto de espigado (DE) resultó superior en el caso de Zeno, por tratarse de un híbrido blanco cultivado como verde (95\%), seguido de DO (4\%), DC (2,6\%) y DP $(0,2 \%)$ (Cuadro 4).

\section{Discusión}

Dada la edad de la plantación, las coronas se encontraban muy cerca de la superficie, lo que motivó que muchos turiones presentaran tendencia al espigado, por lo que la implementación de dos largos de corte hizo posible que, 
Cuadro 3. Distribución por calibres de la producción comercial total (NNT) de turiones cosechados de ocho genotipos de espárrago (Asparagus officinalis var. altilis), en su noveno año productivo (miles de turiones ha-1). Azul, Argentina. 2016.

Table 3. Thickness distribution of the total commercial spear production (NNT) harvested from eight asparagus genotypes (Asparagus officinalis var. altilis) in their ninth production year (thousands of spears ha ${ }^{-1}$ ). Azul, Argentina. 2016.

\begin{tabular}{|c|c|c|c|c|c|c|}
\hline \multirow[b]{2}{*}{ Híbrido } & J (NNT) & XL (NNT) & L (NNT) & M (NNT) & S (NNT) & Asp (NNT) \\
\hline & \multicolumn{6}{|c|}{ Miles turiones ha-1 } \\
\hline Italo & $1,00^{\mathrm{b}}$ & $19,50 \mathrm{bc}$ & 68,48 cde & 79,48 ab & $45,01 \mathrm{bc}$ & 18,00 ab \\
\hline H-668 & $1,50^{\mathrm{b}}$ & $20,50 \mathrm{bc}$ & 82,49 bc & $95,00^{\mathrm{ab}}$ & $64,51^{\mathrm{ab}}$ & $21,00^{a b}$ \\
\hline Zeno & $1,50^{\mathrm{b}}$ & $15,00^{\mathrm{c}}$ & $50,51^{\mathrm{e}}$ & 85,49 ab & $56,00 \mathrm{abc}$ & 18,00 ab \\
\hline Marte & $2,50 \mathrm{ab}$ & $23,50^{\mathrm{bc}}$ & $53,51^{\mathrm{de}}$ & $71,02^{b}$ & $36,00^{c}$ & $7,50^{\mathrm{b}}$ \\
\hline Eros & $2,50^{a b}$ & $30,50^{b}$ & $76,01^{\mathrm{cd}}$ & $87,52^{a b}$ & $65,01^{\mathrm{ab}}$ & $24,50^{\text {a }}$ \\
\hline Ercole & $3,00^{\mathrm{ab}}$ & $27,00 \mathrm{bc}$ & $89,51^{b c}$ & $100,50^{\text {a }}$ & $71,99^{\text {a }}$ & $25,50^{\text {a }}$ \\
\hline UC-157* & $4,50^{\mathrm{ab}}$ & $29,50^{b}$ & $115,52^{\text {a }}$ & 83,50 ab & 45,98 bc & 19,00 ab \\
\hline Giove & $5,50^{\mathrm{a}}$ & $45,51^{\text {a }}$ & $101,52^{a b}$ & 83,50 ab & $61,50 \mathrm{ab}$ & 12,00 ab \\
\hline
\end{tabular}

* testigo / * control.

Cuadro 4. Defectos encontrados en turiones de espárrago verde (Asparagus officinalis var. altilis) de diferentes genotipos (miles de turiones ha-1). Azul, Argentina. 2016.

Table 4. Defects found in green asparagus spears from different green asparagus genotypes (Asparagus officinalis var. altilis). (thousands of spears ha' ${ }^{-1}$ ). Azul, Argentina. 2016.

\begin{tabular}{|c|c|c|c|c|}
\hline \multirow[t]{3}{*}{ Híbridos } & \multicolumn{4}{|c|}{ Turiones defectuosos } \\
\hline & Muy cortos & Espigados & Daño de plagas & Otros defectos \\
\hline & \multicolumn{4}{|c|}{ Miles turiones ha $^{-1}$} \\
\hline Italo & $6,00^{\mathrm{b}}$ & 322,00 def & 0,50 ab & $19,00^{\text {a }}$ \\
\hline H-668 & $9,00^{\mathrm{b}}$ & $401,00 \mathrm{bc}$ & $0,00^{\mathrm{b}}$ & $12,50^{\mathrm{a}}$ \\
\hline Zeno & $6,00^{\mathrm{b}}$ & $489,50^{\text {a }}$ & $0,50^{\mathrm{ab}}$ & $22,00^{\text {a }}$ \\
\hline Marte & 17,00 ab & $449,00^{a b}$ & $0,50^{a b}$ & $18,00^{\text {a }}$ \\
\hline Eros & 13,50 ab & $324,50 \mathrm{de}$ & $1,50 \mathrm{ab}$ & $13,50^{\text {a }}$ \\
\hline Ercole & 8,50 ab & $337,00^{\mathrm{cd}}$ & $0,00^{\mathrm{b}}$ & $18,50^{\text {a }}$ \\
\hline Uc- $157 *$ & 11,50 ab & $256,50^{f}$ & $2,00^{\mathrm{a}}$ & $15,00^{\text {a }}$ \\
\hline Giove & $7,00^{\text {a }}$ & 265,50 ef & 1,00 ab & $12,00^{\text {a }}$ \\
\hline
\end{tabular}

* testigo / * control.

además de la producción comercial tradicional de turiones largos, se pudiera aprovechar una proporción adicional para una categoría gourmet, como lo son las puntas de espárrago, lo cual permite diversificar la oferta y ampliar las posibilidades de mercado.

La producción comercial promedio lograda en el noveno año productivo, de primera calidad, resultó 0,6 t ha-1 superior a la obtenida el año previo (2015), en que también se utilizaron dos largos de corte comercial (Castagnino 
et al., 2016). Por el contrario, en 2014, en que la totalidad de la producción se procesó en un solo largo de corte $(22 \mathrm{~cm}$ ), la misma resultó superior en 1,64 t ha-1 , lo cual estaría indicando que si bien el empleo de ambos largos propicia una mayor cantidad de turiones comerciales, el rendimiento se ve afectado (Castagnino et al., 2015).

La producción comercial lograda mediante los dos largos de corte resultó superior a la reportada por Risso et al. (2012), quienes obtuvieron $3,85 \mathrm{t} \mathrm{ha}^{-1}$ al utilizar como largo de corte comercial $22 \mathrm{~cm}$. Si en el presente estudio solamente se hubiese considerado dicho largo, solo se habría logrado $2,77 \mathrm{t} \mathrm{ha}^{-1}$, y ya que se consideraron los dos largos, se obtuvo un $36 \%$ más de producción. En el caso particular del híbrido Giove, ha venido mejorando su productividad, dado que el primer año evaluado (2008) resultó superado estadísticamente por UC-157, Zeno, Ercole, Eros y H668 (Castagnino et al., 2009), lo cual indicaría una curva productiva más lenta respecto de los restantes genotipos en estudio.

Respecto del testigo, los resultados obtenidos demuestran una buena adaptación regional en plantaciones aún en su décimo año productivo, con una gran dispersión de calibres, posiblemente debido a que el $50 \%$ de las plantas son femeninas, caracterizadas por la producción de turiones de elevado calibre, aunque en menor cantidad, y que el $50 \%$ son masculinos, más productivos en cantidad, pero de menores calibres. Es decir que, en general, dicho genotipo resulta con buena adaptación, solo que por su dispersión de calibres y tipos de turiones (largos y cortos) hace necesario una intensa labor de calibrado durante el procesado, lo cual implica una mayor demora y un mayor costo de acondicionamiento para los emprendedores, y también hace necesario orientar la producción a distintos mercados, acorde a las preferencias de los consumidores.

En número de turiones comerciales se mantuvo la tendencia productiva obtenida en la séptima evaluación (2014) y en los mismos híbridos, a excepción de Giove, quienes superaron a los restantes genotipos (Castagnino et al., 2015).

Respecto a la elevada proporción de turiones con el defecto de espigado, posiblemente se debió a la frecuencia de cosecha utilizada, por lo que convendría que sea diaria. Similares resultados encontraron Castagnino et al. (2017), en una evaluación de la productividad en trece genotipos de espárrago realizada en una plantación iniciada por plantines, en que, con la misma frecuencia de cosecha, el principal defecto fue el de espigado, seguido de otros defectos, como un limitado crecimiento y el daño por plagas. Dichos resultados difirieron de los reportados por Novella et al. (2016), quienes evaluaron la producción de dos años (octavo y noveno año productivos) de una plantación iniciada por el sistema tradicional de arañas o coronas, y obtuvieron $75,2 \%$ de daño por espigado (17,7 $\%$ inferior), lo cual podría indicar una mayor tendencia a este defecto en plantaciones iniciadas con plantines, con cepellón producidos en invernadero, respecto de las raíces o arañas producidas en almácigos a campo de un año, con respecto a las iniciadas mediante el sistema tradicional de arañas. Respecto a la superioridad productiva del genotipo Giove en relación con los restantes híbridos italianos, los resultados obtenidos se corresponden a los logrados por otros autores, quienes, al evaluar el comportamiento invernadero de los genotipos Giove, H-668 y Marte, encontraron que Giove superó a los restantes en el primero (Castagnino et al., 2009), tercero (Risso et al., 2012) y cuarto (Kirschenbilder et al., 2015) año productivo. En dichos estudios, el testigo UC-157 demostró superior comportamiento en el primer y tercer año, siendo superado por los tres masculinos italianos en el cuarto año, y mostró una curva de crecimiento productiva distinta en plantaciones de primicia, más pronunciada inicialmente y más corta que los italianos. Por la naturaleza perecedera de los espárragos se plantea como desafío a futuro la necesidad de complementar el rápido y adecuado procesamiento y envasado, con métodos efectivos para extender su consumo poscosecha con almacenamiento en atmósfera modificada (MAP) (Techavuthiporn y Boonyaritthongchai, 2016). 


\section{Conclusiones}

La productividad comercial, en $\mathrm{kg} \mathrm{ha}^{-1}$, de los genotipos Giove, UC-157 y Ercole resultó superior; mientras que, en número de turiones producidos, además de dichos genotipos también se destacaron Eros y H-668. Cuando el objetivo es la comercialización de turiones de elevado calibre lo apropiado sería cultivar los genotipos Giove y UC-157, como así también cuando se pretende lograr amplia distribución de calibres para abastecer diferentes mercados.

A fin de optimizar el rendimiento comercial y minimizar el defecto de espigado convendría evaluar una frecuencia diaria de cosecha.

El empleo de dos largos de corte permitió incrementar el porcentaje de turiones comerciales y, si bien posiblemente afecte el rendimiento comercial total en $\mathrm{kg}$, permite lograr una proporción adicional acorde a las actuales demandas del mercado de tipos de turiones para ser destinados a uso gourmet.

Dado que el principal defecto encontrado fue el espigado, resulta conveniente incrementar la frecuencia de cosecha a diaria.

\section{Literatura citada}

Albanese, D., L. Russo, A. Cinquanta, A. Brasiello, and M. Di-Matteo. 2007. Physical and chemical changes in minimally processed green asparagus during cold-storage. Food Chem. 101:274-280. doi:10.1016/j.foodchem.2006.01.048

Bhowmik, P.K., T. Matsui, and K. Kawada. 2000. Textural and compositional changes of asparagus spears during storage at 1 ${ }^{\circ} \mathrm{C}$ and subsequent senescence at $25^{\circ} \mathrm{C}$. Pak. J. Biol. Sci. 3:787-790. doi:10.3923/pjbs.2000.787.790

Bhowmik, P.K., T. Matsui, K. Kawada, and H. Suzuki. 2001. Seasonal changes of asparagus spears in relation to enzyme activities and carbohydrate content. Sci. Hort. 88:1-9. doi:10.1016/S0304-4238(00)00199-0

Castagnino, A.M., K.E. Díaz, M.B. Rosini, and A. Falavigna. 2009. Evaluation of some critical points of the asparagus food chain in Argentina. Acta Hortic. 950:37-52. doi:10.17660/ActaHortic.2012.950.3

Castagnino, A.M., K. Diaz, M. Rosini, G. Pizzarro, S. Mondini, G. Martinoia, y A. Falavigna. 2015. Evaluación de la productividad y distribución de calibres de híbridos masculinos italianos de espárrago verde (Asparagus officinalis L.) de una plantación adulta iniciada con dos tamaños de plantines, en Azul. Hortic. Argentina 34(85):66.

Castagnino, A.M., K.E. Diaz, M.B. Rosini, M.B. Tarantino, y A. Guisollis. 2016. Evaluación de la productividad de ocho híbridos de espárrago verde (Asparagus officinalis 1. var. altilis) en su octavo año. Hortic. Argentina 35(88):70.

Castagnino, A.M., L. Fernández, M.B. Rosini, K. Díaz, y W.J. Rogers. 2017. IV Ensayo internacional de cultivares de espárrago en Argentina: factores de cosecha, productividad y comportamiento en poscosecha de una colección de genotipos de espárrago (Asparagus officinalis var. altilis L.). Hortic. Argentina 36(91):46-65.

Chen, X., W. Qin, L. Ma, F. Xu, P. Jin, and Y. Zheng. 2015. Effect of high pressure processing and thermal treatment on physicochemical parameters, antioxidant activity and volatile compounds of green asparagus juice. LWT- Food Sci. Technol. 62:927-933. doi:10.1016/j.lwt.2014.10.068

Di-Rienzo, J.A., F. Casanoves, M.G. Balzarini, L. Gonzalez, M. Tablada, y C.W. Robledo. 2018. InfoStat versión 2018. Grupo InfoStat, FCA, Universidad Nacional de Córdoba, ARG.

Falavigna, A. 2004. Strategie per ottimizzare e valorizzare la produzione di asparago in Sicilia. Programma interregionale di ricerca e sperimentazione applicata, trasferimento delle innovazione agli operatori di filiera e programmi a forte contenuto innovativo. Editorial Grillo e Famá, ITA. 
Falavigna, A. 2006. I punti critici dell’ Asparago. L’Informatore Agrario 1:52-56.

Falavigna, A. 2007. Asparago: resa e qualità col miglioramento genetico. Agricoltura 35:85-87.

FAO, OPS, WFP, y UNICEF. 2018. Panorama de la seguridad alimentaria y nutricional en América Latina y el Caribe 2018. FAO, Roma, ITA. http://www.fao.org/3/CA2127ES/CA2127ES.pdf (consultado 7 marzo 2019).

Kim, B.Y., Z.G. Cui, S.R. Lee, S.J. Kim, H.K. Kang, Y.K. Lee, and D.B. Park. 2009. Effects of Asparagus officinalis extracts on liver cell toxicity and ethanol metabolism. J. Food Sci. 74:H204-H208. doi:10.1111/j.1750-3841.2009.01263.x

Kirschenbilder, E., A.M. Castagnino, K.E. Díaz, M.B. Rosini, y A. Falavigna. 2015. Cadena espárrago: producción de diferentes genotipos en su quinto año y comportamiento en poscosecha. Agron. Mesoam. 26:99-109. doi:10.15517/AM.V26I1.16933

Lill, R.E., G.A. King, and E.M. O’Donoghue. 1990. Physiological changes in asparagus spears immediately after harvest. Sci. Hort. 44:191-199. doi:10.1016/0304-4238(90)90119-Y

Limgroup. 2016. Lim news January 2016. Limgroup, NLD. https:/www.limgroup.eu/download/1312/Downloads/Spaans/ Nieuwsbri even/2016/Webversie_jan2016.pdf (accessed Jul. 10, 2017).

Makris, D.P., and J.T. Rossiter. 2001. Domestic processing of onion bulbs (Allium cepa) and asparagus spears (Asparagus officinalis): effect of flavonol content and antioxidant status. J. Agric. Food Chem. 49:3216-3222. doi:10.1021/jf001497z

Marina, J., A.M. Castagnino, P. Sastre, K. Díaz, y A.P. Guisolis. 2010. Alternativas para optimizar la productividad y asegurar una mejor calidad del espárrago (Asparagus officinalis var. altilis L.). Rev. Colomb. Cienc. Hort. 4:55-66. doi:10.17584/ rcch.2010v4i1.1225

Mastropasqua, L., P. Tanzarella, and C. Paciolla. 2016. Effects of postharvest light spectra on quality and health-related parameters in green Asparagus officinalis L. Postharv. Biol. Technol. 112:143-151. doi:10.1016/j.postharvbio.2015.10.010

MSA (Ministerio de Salud Argentino). s.f. Guías alimentarias de la población Argentina. MSA, ARG. http://www.msal.gob. ar/images/stories/bes/graficos/0000000817cnt-2016-04_Guia_Alimentaria_completa_web.pdf (consultado 5 set. 2018).

OMS, y FAO. 2003. Dieta, nutrición y prevención de enfermedades crónicas. Serie de Informes Técnicos 916. OMS, Ginebra, SUI. https://www.who.int/nutrition/publications/obesity/WHO_TRS_916_spa.pdf (consultado 7 mar. 2019).

Novella, A., A.M. Castagnino, G. Martinoia, M. Durante, K. Diaz, and M.B. Tarantino. 2017. Evaluación productiva y económica de una plantación adulta de espárrago verde Asparagus officinalis var. altilis L. en la provincia de Buenos Aires. Hortic. Argentina 36(89):70-96.

Requesens, E.C. 2011. Bases agroambientales para un desarrollo sustentable del partido de Azul. Universidad Nacional del Centro de la Provincia de Buenos Aires, Buenos Aires, ARG. ftp://www1.faa.unicen.edu.ar/pub/Bases_Agroambientales. pdf (consultado 31 ago. 2018).

Risso, A., A.M. Castagnino, K. Díaz, B.M. Rosini, J. Marina, y A. Falavigna. 2012. Productividad y calidad de cuatro híbridos de espárrago verde (Asparagus officinalis L. var. altilis) en invernadero. Rev. Colom. Cienc. Hort. 6:55-66.

SAGPyA (Secretaría de Agricultura, Ganadería, Pesca y Alimentos) 2007. Protocolo de calidad para espárrago fresco. Resolución SAGPyA No 249/2007. SAGPyA, ARG. http://www.alimentosargentinos.gob.ar/contenido/valorAr/sello/ SAA010_Esparrago_v08.pdf (consultado 22 ago. 2018).

Santos, B. 2011. Análisis económico del empleo de dos híbridos (UC-157 e Italo) de espárrago verde con destino a diferentes mercados. Pontificia Universidad Católica de Argentina, ARG. http://bibliotecadigital.uca.edu.ar/repositorio/tesis/ analisis-economico-empleo-dos-hibridos.pdf (consultado 22 ago. 2018).

Snededor, G.W., and W.G. Cochran. 1980. Statistical methods. 7th ed. The Iowa State University Press, AI, USA. 
Soobrattee, M.A., V.S. Neergheen, A. Luximon-Ramma, O.I. Aruoma, and T. Bahorun. 2005. Phenolics as potential antioxidant therapeutic agents: mechanism and actions. Mutation Res. 579:200-213. doi:10.1016/j.mrfmmm.2005.03.023

Sun, T., J.R. Powers, and J. Tang. 2007a. Evaluation of the antioxidant activity of asparagus, broccoli and their juices. Food Chem. 105:101-106. doi:10.1016/j.foodchem.2007.03.048

Techavuthiporn, C., and P. Boonyaritthongchai. 2016. Effect of prestorage short-term anoxia treatment and modified atmosphere packaging on the physical and chemical changes of green asparagus. Postharv. Biol. Technol. 117:64-70. doi:10.1016/j. postharvbio.2016.01.016

Vázquez-Rowe, I., R. Kahhat, I. Quispe, and M. Bentín. 2016. Environmental profile of green asparagus production in a hyperarid zone in coastal Peru. J. Clean. Prod. 112:2505-2517. doi:10.1016/j.jclepro.2015.09.076

Wang, B.S., L.W. Chang, H.C. Wu, S.L. Huang, H.L. Chu, and M.H. Huang. 2011. Antioxidant and antityrosinase activity of aqueous extracts of green asparagus. Food. Chem. 127:141-146. doi:10.1016/j.foodchem.2010.12.102 